\title{
TWO-GENERATOR GROUPS WITH PERFECT FRATTINI SUBGROUPS
}

\author{
M. J. EVANS
}

\begin{abstract}
In this paper, 2-generator groups with perfect Frattini subgroups are constructed. The construction also shows that every countable group can be embedded in the Frattini subgroup of a 2-generator group.
\end{abstract}

1. Introduction. It is well-known that the Frattini subgroup $\Phi(G)$ of a finite group $G$ is nilpotent. However, for infinite groups the situation is considerably more complicated. For instance, in [1] P. Hall constructed infinite 2-generator soluble groups with non-nilpotent Frattini subgroups. More recently, M. F. Newman (unpublished) has constructed finitely generated, infinite $p$-groups with insoluble Frattini subgroups.

In this note we use a construction similar to Hall's (loc. cit.) to obtain 2-generator groups with perfect Frattini subgroups. More precisely, we prove

THEOREM 1.1. Let $K$ be a countable perfect group. Then there exists a 2generator group $G_{K}$, such that $\Phi\left(G_{K}\right)$ is a direct product of $\aleph_{0}$ copies of $K$.

Since each countable group can be embedded in a countable, nonabelian, simple (and therefore perfect) group (e.g. [2, Theorem 3.4]), Theorem 1.1 implies the following.

COROLLARY 1.2. Every countable group can be embedded in the Frattini subgroup of a 2-generator group.

2. The construction. Throughout the sequel $M$ denotes a countable perfect group of infinite exponent.

Suppose $M=\left\{m_{1}, m_{2}, m_{3}, \ldots\right\}$ and let $S$ denote the sequence $m_{1}, m_{2}, m_{1}, m_{2}$, $m_{3}, m_{1}, m_{2}, m_{3}, m_{4}, m_{1}, \ldots, m_{i}, m_{1}, \ldots, m_{i}, m_{i+1}, m_{1}, \ldots$. Write $S_{j}$ for the $j$ th term of $S$.

REMARK. Note that for any integer $N$ and any $m \in M$ there exists an integer $n>N$ such that $S_{n}=m$. This fact will be crucial in the proof of Lemma 3.1.

Let $C=\mathrm{Cr}_{i \in \mathbf{Z}} M_{i}$ and $H=\operatorname{Dr}_{i \in \mathbf{Z}} M_{i}$, where $M_{i} \simeq M$ for all $i \in \mathbf{Z}$. Thus $C$ is the cartesian product and $H$ the direct product of $\aleph_{0}$ copies of $M$. Given any $g \in C$ we write $(g)_{i}$ for the $i$ th coordinate of $g$ and define the support of $g$ to be the set $\sigma(g)=\left\{i \mid(g)_{i} \neq 1\right\}$. Clearly $g \in H$ if and only if $\sigma(g)$ is a finite set.

Let $z$ generate an infinite cyclic group that is disjoint from $C$ and define an action of $\langle z\rangle$ on $C$ by

$$
\left(g^{z}\right)_{i+1}=(g)_{i}, \quad \text { for all } g \in C \text { and } i \in \mathbf{Z} .
$$

Define $C^{*}$ to be the split extension of $C$ by $\langle z\rangle$.

Received by the editors March 6, 1986.

1980 Mathematics Subject Classification (1985 Revision). Primary 20F26, $20 \mathrm{E} 28$. 
Let $w \in C$ be the element such that

$$
\begin{aligned}
(w)_{2^{j}}=S_{j} & \text { if } j \geq 1 \\
(w)_{l}=1 & \text { if } l \neq 2^{j} \text { for all } j \geq 1 .
\end{aligned}
$$

Finally, define $G_{M}=\langle H, w, z\rangle$, a subgroup of $C^{*}$. Observe that $M_{i}^{z}=M_{i+1}$ and $M_{i}^{w}=M_{i}$, for all $i \in \mathbf{Z}$, so $H \triangleleft G_{M}$.

The notation introduced above will be used for the remainder of this note. We shall show that $\Phi\left(G_{M}\right)=H$ and use this fact to deduce Theorem 1.1.

3. The lemmas. Our main lemma is the following.

LEMMA 3.1. Let $h_{1}$ and $h_{2}$ be arbitrary elements of $H$. Then $G_{M}=\left\langle h_{1} w, h_{2} z\right\rangle$.

PROOF. Since $\sigma\left(h_{1}\right)$ and $\sigma\left(h_{2}\right)$ are finite sets, there exists an integer $N>0$ such that $\left(h_{1} w\right)_{n}=(w)_{n}$ and $\left(h_{2}\right)_{n}=1$ for all $n \geq N$. We consider such an $N$ fixed once for all and define $p$ to be the least integer such that $2^{p} \geq N$. We shall prove that $M_{2^{p}} \leq\left\langle h_{1} w, h_{2} z\right\rangle$. Since $\left(h_{2} z\right)^{-k} M_{2^{p}}\left(h_{2} z\right)^{k}=M_{2^{p}+k}$, for all $k \in \mathbf{Z}$, and $H=\operatorname{Dr}_{i \in \mathbf{Z}} M_{i}$, the result then follows immediately.

As $M$ is perfect, it suffices to show that, given arbitrary elements $m_{k}, m_{j} \in M$, there exists $y \in\left\langle h_{1} w, h_{2} z\right\rangle \cap H$ such that

$$
(y)_{2^{p}}=\left[m_{k}, m_{j}\right]
$$

and

$$
(y)_{l}=1 \text { if } l \neq 2^{p} .
$$

Suppose that $m_{k}$ and $m_{j}$ are given elements of $M$ and let $t$ denote the least integer in $\sigma\left(h_{1} w\right)$. It follows from the remark in $\S 2$ that there exists an integer $r>p$ such that $S_{r}=m_{k}$. Furthermore, there exists an integer $u>r$ such that $S_{u}=m_{j}$ and $2^{r}-t<2^{u-1}$. The letters $r$ and $u$ retain this meaning throughout.

Define $\lambda_{1}, \lambda_{2} \in\left\langle h_{1} w, h_{2} z\right\rangle$ by $\lambda_{i}=\left(h_{2} z\right)^{q_{i}} h_{1} w\left(h_{2} z\right)^{-q_{i}}$, where $q_{1}=2^{r}-2^{p}$ and $q_{2}=2^{u}-2^{p}$. We claim that $y=\left[\lambda_{1}, \lambda_{2}\right]$ is the desired element satisfying $(1)$ and (2).

Bearing in mind that $q_{i}>0$, for $i=1,2$, we have

$$
\left(h_{2} z\right)^{q_{i}}=h_{2} h_{2}^{z^{-1}} \cdots h_{2}^{z^{-\left(q_{i}-1\right)}} z^{q_{i}}=f_{i} z^{q_{i}}, \text { say, }
$$

where $f_{i} \in H$. Thus $\left(\lambda_{i}\right)_{2^{p}}=\left(f_{i}\right)_{2^{p}}\left(\left(h_{1} w\right)^{z^{-q_{i}}}\right)_{2^{p}}\left(f_{i}^{-1}\right)_{2^{p}}$. However, $f_{i}=$ $\prod_{a=0}^{q_{i}-1} h_{2}^{z^{-a}}$ so

$$
\left(f_{i}\right)_{2^{p}}=\prod_{a=0}^{q_{i}-1}\left(h_{2}^{z^{-a}}\right)_{2^{p}}=\prod_{a=0}^{q_{i}-1}\left(h_{2}\right)_{2^{p}+a}
$$

for $i=1,2$. Now $2^{p}+a \geq N$ for $a=0,1, \ldots, q_{i}-1$ and, by our choice of $N$, $\left(h_{2}\right)_{n}=1$ for all $n \geq N$. Hence $\left(f_{i}\right)_{2^{p}}=1$ for $i=1,2$. Therefore $\left(\lambda_{i}\right)_{2^{p}}=$ $\left(\left(h_{1} w\right)^{z^{-q_{i}}}\right)_{2^{p}}=\left(h_{1} w\right)_{2^{p}+q_{i}}$ for $i=1,2$. Recall that $\left(h_{1} w\right)_{n}=(w)_{n}$ for all $n \geq N$. It follows that $\left(\lambda_{1}\right)_{2^{p}}=\left(h_{1} w\right)_{2^{r}}=(w)_{2^{r}}=S_{r}=m_{k}$ by our choice of $r$, and similarly $\left(\lambda_{2}\right)_{2^{p}}=\left(h_{1} w\right)_{2^{u}}=S_{u}=m_{j}$. Hence $\left(\left[\lambda_{1}, \lambda_{2}\right]\right)_{2^{p}}=\left[\left(\lambda_{1}\right)_{2^{p}},\left(\lambda_{2}\right)_{2^{p}}\right]=$ $\left[m_{k}, m_{j}\right]$ and $y=\left[\lambda_{1}, \lambda_{2}\right]$ satisfies $(1)$.

We next show that $\sigma\left(\lambda_{1}\right) \cap \sigma\left(\lambda_{2}\right) \subseteq\left\{2^{p}\right\}$. Suppose that $b \in \sigma\left(\lambda_{1}\right) \cap \sigma\left(\lambda_{2}\right)$ and observe that $\sigma\left(\lambda_{i}\right)=\left\{v-q_{i} \mid v \in \sigma\left(h_{1} w\right)\right\}$, for $i=1$, 2. It follows that $b=v_{1}-q_{1}=$ 
$v_{2}-q_{2}$ where $v_{1}, v_{2} \in \sigma\left(h_{1} w\right)$. Therefore $v_{2}=v_{1}-q_{1}+q_{2}=v_{1}-2^{r}+2^{u}$ and as $t$ is the least integer in $\sigma\left(h_{1} w\right)$ this implies that $v_{2} \geq t-2^{r}+2^{u}$. However $2^{r}-t<2^{u-1}$, so $v_{2}>2^{u-1} \geq N$. It follows from our choice of $N$ and the fact that $v_{2} \in \sigma\left(h_{1} w\right)$ that $v_{2}=2^{n_{2}}$ for some integer $n_{2} \geq u$. Now $v_{1}=2^{n_{2}}+q_{1}-q_{2}=2^{n_{2}}+2^{r}-2^{u} \geq$ $2^{r} \geq N$ so $v_{1}=2^{n_{1}}$ for some integer $n_{1} \geq r$. Hence $b=2^{n_{1}}+2^{p}-2^{r}=2^{n_{2}}+2^{p}-2^{u}$ so $2^{r}\left(2^{n_{1}-r}-1\right)=2^{r}\left(2^{n_{2}-r}-2^{u-r}\right)$ which implies that $n_{1}=r$ and $n_{2}=u$. Thus $b=2^{p}$ and $\sigma\left(\lambda_{1}\right) \cap \sigma\left(\lambda_{2}\right) \subseteq\left\{2^{p}\right\}$.

Note that $\sigma\left(\left[\lambda_{1}, \lambda_{2}\right]\right) \subseteq \sigma\left(\lambda_{1}\right) \cap \sigma\left(\lambda_{2}\right)$ so $\left(\left[\lambda_{1}, \lambda_{2}\right]\right)_{l}=1$ if $l \neq 2^{p}$ and $y=\left[\lambda_{1}, \lambda_{2}\right]$ satisfies (2). The proof is complete.

Lemma 3.1 has the following easy consequence.

LEMMA 3.2. The subgroup $H$ of $G_{M}$ is omissible.

Proof. Suppose $G_{M}=\langle H, X\rangle$ where $X$ is a subset of $G_{M}$. Define $N=\langle X\rangle$ so that $G=H N$. Now there exists $h_{1}, h_{2} \in H$ and $n_{1}, n_{2} \in N$ such that $w=h_{1}^{-1} n_{1}$ and $z=h_{2}^{-1} n_{2}$. Thus $h_{1} w, h_{2} z \in N$, so $N=G_{M}$, by Lemma 3.1. Therefore $N=G_{M}=\langle X\rangle$, as required.

Of course, Lemma 3.2 implies that $H \leq \Phi\left(G_{M}\right)$.

Our present goal, as mentioned at the end of $\S 2$, is to show that $\Phi\left(G_{M}\right)=H$. We have established that $\Phi\left(G_{M}\right) \geq H$, so it is enough to prove that $\Phi\left(G_{M} / H\right)=1$. A routine argument, along the lines of $[\mathbf{1}$, Theorem 7$]$, and using the fact that $M$ is of infinite exponent, shows that $G_{M} / H \simeq \mathbf{Z}$ wr $\mathbf{Z}$, the restricted wreath product of two infinite cyclic groups. Moreover, it is easily seen that the Frattini subgroup of $\mathbf{Z}$ wr $\mathbf{Z}$ is trivial. Summing up, we have

LEMMA 3.3. Let $M$ be a countable perfect group of infinite exponent and let $G_{M}$ be the group constructed above. Then $\Phi\left(G_{M}\right)=H$, a direct product of $\aleph_{0}$ copies of $M$.

4. Proof of Theorem 1.1. If $K$ is of infinite exponent the result follows by Lemma 3.3, so suppose $K$ is of finite exponent.

Let $N$ be a countable perfect group of infinite exponent and define $M=K \times N$, a countable perfect group of infinite exponent. Consider the group $G_{M}$ constructed above. By Lemma 3.3 we have $\Phi\left(G_{M}\right)=H=\operatorname{Dr}_{i \in \mathbf{Z}} M_{i}$, where $M_{i} \simeq M$ and $M_{i}^{z^{j}}=M_{i+j}$, for all $i, j \in \mathbf{Z}$.

We may write $M_{0}$ as a direct product $M_{0}=K_{0} \times N_{0}$, where $K_{0} \simeq K$ and $N_{0} \simeq N$. Let $K_{i}=K_{0}^{z^{i}}, N_{i}=N_{0}^{z^{i}}$, and notice that $M_{i}=K_{i} \times N_{i}, N_{i}^{z}=N_{i+1}$, and $N_{i}^{w}=N_{i}$, for all $i \in \mathbf{Z}$. Lemma 3.1 shows that $G_{M}=\langle w, z\rangle$ and it follows that $D=\operatorname{Dr}_{i \in \mathbf{Z}} N_{i}$ is a normal subgroup of $G_{M}$.

Clearly $H / D \simeq \operatorname{Dr}_{i \in \mathbf{Z}} K_{i}$ and the result follows on setting $G_{K}=G_{M} / D$.

ACKNOWLEDGMENTS. The results presented here are contained in Chapter V of my Ph.D. thesis. I would like to record my gratitude to my supervisor, James Wiegold, who gave me much help and encouragement during my time as a research student. I am also indebted to the University of Wales for providing me with financial support. 


\section{REFERENCES}

1. P. Hall The Frattini subgroups of finitely generated groups, Proc. London Math. Soc. (3) 11 (1961), 327-352.

2. R. C. Lyndon and P. E. Schupp, Combinatorial group theory, Springer-Verlag, Berlin-Heidelberg-New York, 1977.

Department of Mathematics, University of Alabama, University, Alabama 35486 